\title{
Functional equations for exponential polynomials
}

\author{
ŻYWILla FECHNER(D AND LÁSZLÓ SzÉKELYHIDI(D
}

\begin{abstract}
The aim of the present paper is to describe some properties of functions with finite dimensional difference spaces by means of spectral analysis and spectral synthesis. We are going to apply these results to a version of a Levi-Civita functional equation, which has been recently studied by J. M. Almira and E. Shulman.
\end{abstract}

Mathematics Subject Classification. 39B99, 43A62, 20N20.

Keywords. Exponential polynomials, Levi-Civita equation, Hypergroups.

\section{Introduction}

Let $H$ be a commutative discrete hypergroup with convolution $*$, involution $~$, and identity $o$ (the definition and comprehensive studies on hypergroup theory can be found in [4]). Let $\mathcal{C}(H)$, respectively $\mathbb{C} H$ denote the set of all complex valued functions, respectively the set of finitely supported complex valued functions on $H$. Equipped with pointwise linear operations (addition and multiplication with complex scalars) and with the topology of pointwise convergence $\mathcal{C}(H)$ is a locally convex topological vector space. The topological dual of $\mathcal{C}(H)$ can be identified with $\mathbb{C} H$ using the obvious pairing

$$
\langle\mu, f\rangle=\sum_{x} f(x) \mu(x)
$$

for $\mu$ in $\mathbb{C} H$ and $f$ in $\mathcal{C}(H)$, where the sum ranges over all elements of $H$. On the space $\mathbb{C} H$ we introduce convolution by the formula

$$
\langle\mu * \nu, f\rangle=\sum_{x} \sum_{y} f(x * y) \mu(x) \nu(y)
$$

for each $f$ in $\mathcal{C}(H)$, whenever $\mu, \nu$ are in $\mathbb{C} H$. Here the sums range over all elements of $H$. Then $\mathbb{C} H$ is a commutative complex algebra with unit $\delta_{o}$, the characteristic function of the singleton $\{o\}$. In general, $\delta_{x}$ denotes the 
characteristic function of the singleton $\{x\}$ for each $x$ in $H$. We call $\mathbb{C} H$ the hypergroup algebra of $H$. The convolution

$$
\mu * f(x)=\sum_{y} f(x * \check{y}) \mu(y)
$$

for $\mu$ in $\mathbb{C} H, f$ in $\mathcal{C}(H)$ and $x$ in $H$ makes $\mathcal{C}(H)$ a topological module over the hypergroup algebra. The closed submodules of this module are called varieties. It is clear that a linear subspace $X$ of $\mathcal{C}(H)$ is a variety if and only if it is translation invariant, that is, for each $f$ in $X$ and for each $y$ in $H$ we have that $\delta_{y} * f$ is in $X$. For obvious reasons, $\delta_{\breve{y}} * f$ is called the translate of $f$ by $y$. The smallest variety containing the function $f$ is called the variety of $f$ and is denoted by $\tau(f)$. It is easy to see that one dimensional varieties are exactly the varieties of exponentials. We recall that $m: H \rightarrow \mathbb{C}$ is an exponential if it is non-identically zero and satisfies $m(x * y)=m(x) m(y)$. Then we have $m(o)=1$. We say that spectral analysis holds for a variety $V$ if every nonzero subvariety of $V$ contains an exponential. In [13] it was proved that spectral analysis holds for every finite dimensional variety.

We define exponential monomials on $H$ using modified differences as follows (see $[8,9])$. Let $m: H \rightarrow \mathbb{C}$ be an exponential, $y$ an element in $H$ and

$$
\Delta_{m ; y}=\delta_{\check{y}}-m(y) \delta_{o} .
$$

Then $\Delta_{m ; y}$ is in $\mathbb{C} H$ and we call it the modified difference associated with $m$ with increment $y$. The iterates of modified differences are denoted as follows:

$$
\Delta_{m ; y_{1}, y_{2}, \ldots, y_{n}}=\Pi_{k=1}^{n} \Delta_{m ; y_{k}}=\Delta_{m ; y_{n}} * \cdots * \Delta_{m ; y_{1}},
$$

i.e., the right side is a convolution product. If $y_{1}=y_{2}=\cdots=y_{n}=y$ then we simply write $\Delta_{m ; y}^{n}$ for this product. The function $f: H \rightarrow \mathbb{C}$ is called an exponential monomial if $\tau(f)$ is finite dimensional and there is an exponential $m$ and a natural number $n$ such that

$$
\Delta_{m ; y_{1}, y_{2}, \ldots, y_{n+1}} * f=0
$$

for each $y_{1}, y_{2}, \ldots, y_{n+1}$ in $H$. In general, if $f$ satisfies the previous condition, but $\tau(f)$ is not necessarily of finite dimension, then we call $f$ a generalized exponential monomial. It can be shown that if $f$ is nonzero, then $m$ is unique and then we say that $f$ is associated with $\mathrm{m}$. In this case the smallest natural number with the above property is called the degree of $f$. The zero function is an exponential monomial associated with every exponential, and we do not assign a degree to it. Linear combinations of exponential monomials are called exponential polynomials and linear combinations of generalized exponential monomials are called generalized exponential polynomials. We say that a variety $V$ is synthesizable if all exponential monomials in $V$ span a dense subspace. If every subvariety of $V$ is synthesizable, then we say that spectral synthesis holds for $V$. It is known that if a nonzero exponential monomial associated with the exponential $m$ belongs to a variety, then $m$ itself belongs to that variety. 
Consequently, spectral synthesis for a variety implies spectral analysis, but the converse is not necessarily true. For more details on spectral analysis and synthesis on groups see [10, Chapters 11-15]. In [14, Theorem 3] it was proved that spectral synthesis holds for every finite dimensional variety. In fact, every finite dimensional variety consists of exponential polynomials.

Generalized exponential polynomials form a basic function class in the theory of functional equations. The solution space of a large number of classical equations consists of generalized exponential polynomials. There is an extensive literature about this function class and about different characterizations of these functions. Some recent results can be found in [1,2] and the references therein. In [3], the authors prove some new properties of exponential polynomials. In the present paper we join this research by proving further results concerning properties of generalized exponential polynomials on hypergroups.

\section{Functions with finite dimensional difference spaces}

In this section we study functions on commutative discrete hypergroups with the property that all their iterated modified differences of some degree belong to some finite dimensional linear space. Let $H$ be a commutative discrete hypergroup and suppose that the function $f: H \rightarrow \mathbb{C}$ has the property that there is an exponential $m$, a natural number $n$ and a finite dimensional variety $W$ in $\mathcal{C}(H)$ such that $\Delta_{m ; y_{1}, y_{2}, \ldots, y_{n+1}} * f$ is in $W$ for each $y_{1}, y_{2}, \ldots, y_{n+1}$ in $H$. What can we say about $f$ ? Is it true that $f$ is an exponential polynomial? By spectral synthesis on finite dimensional varieties, $W$ consists of exponential polynomials. By finite dimensionality, we might expect that there are finitely many exponentials such that all exponential polynomials in $W$ are associated with these exponentials, and in addition, the degrees of all exponential monomials in $W$ are bounded above. This suggests that $f$ itself must be an exponential polynomial - possibly generalized. In fact, we shall prove this statement below, even for the case where $W$ is replaced by a finite dimensional subspace which is not necessarily a variety.

Lemma 1. Let $W$ be a d-dimensional variety in $\mathcal{C}(H)$. Then there exists a natural number $s$ and there exist different exponentials $m_{1}^{\prime}, m_{2}^{\prime}, \ldots, m_{s}^{\prime}$ on $H$ and natural numbers $l_{1}, l_{2}, \ldots, l_{s}$ such that $l_{1}+l_{2}+\cdots+l_{s}+s \leq d$, and

$\Delta_{m_{1}^{\prime} ; y_{1,1}, y_{1,2}, \ldots, y_{1, l_{1}+1}} * \Delta_{m_{2}^{\prime} ; y_{2,1}, y_{2,2}, \ldots, y_{2, l_{2}+1}} * \cdots * \Delta_{m_{s}^{\prime} ; y_{s, 1}, y_{s, 2}, \ldots, y_{s, l_{s}+1}} * f=0$ holds for each $f$ in $W$ and $y_{j, 1}, y_{j, 2}, \ldots, y_{j, l_{j}+1}$ in $H(j=1,2, \ldots, s)$.

Proof. By spectral synthesis on finite dimensional varieties (see [14]), $W$ has a basis consisting of exponential monomials. In other words, there exist a natural number $s$, different exponentials $m_{1}^{\prime}, m_{2}^{\prime}, \ldots, m_{s}^{\prime}$ on $H$, natural numbers 
$l_{1}, l_{2}, \ldots, l_{s}$ and a basis $\varphi_{1}, \varphi_{2}, \ldots, \varphi_{d}$ of $W$ consisting of exponential monomials associated with the $m_{j}$ 's such that

$$
\Delta_{m_{j}^{\prime} ; y_{j, 1}, y_{j, 2}, \ldots, y_{j, l_{j}+1}} * \varphi_{j}=0 \text { for } j=1,2, \ldots, d .
$$

It follows that any linear combination of the functions $\varphi_{j}$ is annihilated by

$$
\Delta_{m_{1}^{\prime} ; y_{1,1}, y_{1,2}, \ldots, y_{1, l_{1}+1}} * \Delta_{m_{2}^{\prime} ; y_{2,1}, y_{2,2}, \ldots, y_{2, l_{2}+1}} * \cdots * \Delta_{m_{s}^{\prime} ; y_{s, 1}, y_{s, 2}, \ldots, y_{s, l_{s}+1}}
$$

whenever $y_{j, 1}, y_{j, 2}, \ldots, y_{j, l_{j}+1}$ is in $H$ for $j=1,2, \ldots, s$. As the $\varphi_{j}$ 's form a basis of $W$ our statement follows.

Before we formulate the next result it will be convenient to generalize modified differences. For each $\mu$ in $\mathbb{C} H$ and exponential $m: H \rightarrow \mathbb{C}$ we introduce the notation

$$
\hat{\mu}(m)=\langle\mu, \check{m}\rangle .
$$

The function $\widehat{\mu}$, which is defined on the set of all exponentials is the FourierLaplace transform of $\mu$ (see [4]). Then for each $\mu$ in $\mathbb{C} H$ and exponential $m$ we define

$$
\Delta_{m ; \mu}=\breve{\mu}-\widehat{\mu}(\check{m}) \delta_{o} .
$$

This is the generalization of $\Delta_{m ; y}$ as for any exponential $m: H \rightarrow \mathbb{C}$ we have

$$
\Delta_{m ; y}=\Delta_{m ; \delta_{y}}
$$

for all $y$ in $H$. Indeed, let $m: H \rightarrow \mathbb{C}$ be an arbitrary exponential. We have

$$
\begin{aligned}
\Delta_{m ; \delta_{y}} & =\check{\delta_{y}}-\widehat{\delta_{y}}(\check{m}) \delta_{o} \\
& =\delta_{\breve{y}}-\delta_{y}(m) \delta_{o}=\delta_{\breve{y}}-m(y) \delta_{o}=\Delta_{m ; y}
\end{aligned}
$$

for all $y$ in $H$.

Observe that formula (1) can be written more explicitly: using the fact that $\widehat{\mu}(\check{m})=\mu(m)$ for an arbitrary $f$ in $\mathcal{C}(H)$ we have

$$
\begin{aligned}
\Delta_{m ; \mu} * f(x) & =\breve{\mu} * f(x)-\widehat{\mu}(\breve{m}) f(x) \\
& =\sum_{y} f(x * y) \mu(y)-f(x) \sum_{y} m(y) \mu(y) \\
& =\sum_{y}(f(x * y)-m(y) f(x)) \mu(y)
\end{aligned}
$$

for all $x$ and $y$ in $H$. In particular, using the obvious notation $\Delta_{m ; y * z}=\Delta_{m ; \delta_{y * z}}$ we can write

$$
\Delta_{m ; y * z} * f(x)=\delta_{\breve{y}} * \delta_{\check{z}} * f(x)-m(y * z) f(x)=f(x * y * z)-m(y) m(z) f(x)
$$

for all $x, y, z$ in $H$. We shall use this below. 
Theorem 1. Let $d, k$ be natural numbers, $k>0, V$ a d-dimensional linear subspace in $\mathcal{C}(H), m_{1}, m_{2}, \ldots, m_{k}$ different exponentials on $H, n_{1}, n_{2}, \ldots, n_{k}$ natural numbers, and $f$ in $\mathcal{C}(H)$ such that

$\Delta_{m_{1} ; y_{1,1}, y_{1,2}, \ldots, y_{1, n_{1}+1}} * \Delta_{m_{2} ; y_{2,1}, y_{2,2}, \ldots, y_{2, n_{2}+1}} * \cdots * \Delta_{m_{k} ; y_{k, 1}, y_{k, 2}, \ldots, y_{k, n_{k}+1}} * f$

is in $V$ for each $y_{j, 1}, y_{j, 2}, \ldots, y_{k, n_{k}+1}$ in $H$. Then $f$ is a generalized exponential polymonomial of degree at most $d+n_{1}+\cdots+n_{k}+k$.

Proof. As every element of $\mathbb{C} H$ is a finite linear combination of $\delta$ 's we infer that

$\Delta_{m_{1}, \mu_{1,1}, \mu_{1,2}, \ldots, \mu_{1, n_{1}+1}} * \Delta_{m_{2}, \mu_{2,1}, \mu_{2,2}, \ldots, \mu_{2, n_{2}+1}} * \cdots * \Delta_{m_{k}, \mu_{1,1}, \mu_{1,2}, \ldots, \mu_{1, n_{k}+1}} * f$

is in $V$ for each $\mu_{j, 1}, \mu_{j, 2}, \ldots, \mu_{j, n_{j}+1}$ in $\mathbb{C} H(j=1,2, \ldots, k)$. Let $W$ be the linear span of all these functions with $\mu_{j, 1}, \mu_{j, 2}, \ldots, \mu_{j, n_{j}+1}$ in $\mathbb{C} H(j=$ $1,2, \ldots, k)$; then $W$ is a linear subspace of $V$. We show that $W$ is translation invariant. It is enough to show that all translates of the functions

$\Delta_{m_{1} ; y_{1,1}, y_{1,2}, \ldots, y_{1, n_{1}+1}} * \Delta_{m_{2} ; y_{2,1}, y_{2,2}, \ldots, y_{2, n_{2}+1}} * \cdots * \Delta_{m_{k} ; y_{k, 1}, y_{k, 2}, \ldots, y_{k, n_{k}+1}} * f$

belong to $W$. But this follows from the obvious identity

$$
\begin{aligned}
\delta_{\check{z}} *\left(\Delta_{m ; y} * f\right)(x)= & f(x * y * z)-m(y) f(x * z) \\
= & f(x * y * z)-m(y * z) f(x)+m(y) m(z) f(x) \\
& -m(y) f(x * z) \\
= & \left(\Delta_{m ; y * z} * f\right)(x)-m(y)\left(\Delta_{m ; z} * f\right)(x),
\end{aligned}
$$

which holds for every exponential $m$ and for each $x, y, z$ in $H$. Hence $W$ is a finite dimensional variety in $V$. It is clear (see [14]) that it has a basis consisting of exponential monomials $\varphi_{1}, \varphi_{2}, \ldots, \varphi_{s}$ of degree at most $l_{j}$ associated with the different exponentials $m_{j}^{\prime}(j=1,2, \ldots, s)$. Obviously, here $l_{1}+l_{2}+\cdots+$ $l_{s}+s \leq d$. By the previous theorem, it follows that

$$
\Delta_{m_{1}^{\prime} ; z_{1,1}, z_{1,2}, \ldots, z_{1, l_{1}+1}} * \cdots * \Delta_{m_{s}^{\prime} ; z_{s, 1}, z_{s, 2}, \ldots, z_{s, l_{s}+1}} * \varphi=0
$$

holds for each $\varphi$ in $W$ and $z_{1}, z_{2}, \ldots, z_{k}$ in $H$ (see also [11]). In particular, we have for each $y_{1}, y_{2}, \ldots, y_{k}$ in $H$

$$
\begin{array}{r}
\Delta_{m_{1}^{\prime} ; z_{1,1}, z_{1,2} \ldots, z_{1, l_{1}+1}} * \cdots * \Delta_{m_{s}^{\prime} ; z_{s, 1}, z_{s, 2}, \ldots, z_{s, l_{s}+1}} * \Delta_{m_{1} ; y_{1,1}, y_{1,2} \ldots, y_{1, n_{1}+1}} * \cdots \\
\cdots * \Delta_{m_{k} ; y_{k, 1}, y_{k, 2}, \ldots, y_{k, n_{k}+1}} * f=0 .
\end{array}
$$

Let $M_{i}^{\prime}$, respectively $M_{j}$, denote the ideal in $\mathbb{C} H$ generated by the measures $\Delta_{m_{i}^{\prime}, y}$ for $i=1,2, \ldots, s$, respectively $\Delta_{m_{j}, y}$, for $j=1,2, \ldots, k$ whenever $y$ is in $H$. Then the previous equation implies that

$$
\Pi_{i=1}^{s} M_{i}^{l_{i}+1} \cdot \Pi_{j=1}^{k} M_{j}^{n_{j}+1} \subseteq \operatorname{Ann} \tau(f),
$$


where Ann $\tau(f)$ denotes the annihilator ideal of the variety $\tau(f)$ in $\mathbb{C} H$. It follows from Theorem 4.1 in [9], that $f$ is a generalized exponential polynomial. The given estimate on the degree is an easy consequence of the above computations.

Obviously, in the condition of the previous theorem there are too many free variables. It is reasonable to ask if we can decrease the number of those variables so that the statement remains valid. In the case of commutative semigroups we have the following stronger result. We note that our previous result obviously holds for any commutative semigroup with identity where the involution is meant to be the identity mapping.

Theorem 2. Let $H$ be a commutative semigroup with identity. Let $d, k$ be natural numbers with $k>0, V$ a d-dimensional linear subspace in $\mathcal{C}(H)$, $m_{1}, m_{2}, \ldots, m_{k}$ different exponentials on $H, n_{1}, n_{2}, \ldots, n_{k}$ natural numbers, and $f$ in $\mathcal{C}(H)$ such that

$$
\Delta_{m_{1} ; y_{1}}^{n_{1}+1} * \Delta_{m_{2} ; y_{2}}^{n_{2}+1} * \cdots * \Delta_{m_{k} ; y_{k}}^{n_{k}+1} * f
$$

is in $V$ for each $y_{1}, y_{2}, \ldots, y_{k}$ in $H$. Then $f$ is a generalized exponential polymonomial of degree at most $d+n_{1}+\cdots+n_{k}+k$.

Proof. We follow the ideas applied in the proof of the previous theorem. Exactly in the same way as we did in the previous proof we define $W$ as the linear span of all functions of the form

$$
\Delta_{m_{1} ; \mu_{1}}^{n_{1}+1} * \Delta_{m_{2} ; \mu_{2}}^{n_{2}+1} * \cdots * \Delta_{m_{k} ; \mu_{k}}^{n_{k}+1} * f
$$

with $\mu_{1}, \mu_{2}, \ldots, \mu_{k}$ in $\mathbb{C} H$. Then $W$ is a variety in $V$, in particular, it is finite dimensional. Let $W$ have a basis consisting of the exponential monomials $\varphi_{1}, \varphi_{2}, \ldots, \varphi_{s}$ of degree at most $l_{j}$ associated with the different exponentials $m_{j}^{\prime}(j=1,2, \ldots, s)$. Obviously, here $l_{1}+l_{2}+\cdots+l_{s}+s \leq d$. It follows that

$$
\Delta_{m_{1}^{\prime} ; z_{1,1}, z_{1,2}, \ldots, z_{1, l_{1}+1}} * \cdots * \Delta_{m_{s}^{\prime} ; z_{s, 1}, z_{s, 2}, \ldots, z_{s, l_{s}+1}} * \varphi=0
$$

holds for each $\varphi$ in $W$ and $z_{1}, z_{2}, \ldots, z_{k}$ in $H$. In particular, we have

$$
\Delta_{m_{1}^{\prime} ; z_{1}}^{l_{1}+1} * \Delta_{m_{2}^{\prime} ; z_{2}}^{l_{2}+1} * \cdots * \Delta_{m_{s}^{\prime} ; z_{s}}^{l_{s}+1} * \Delta_{m_{1} ; y_{1}}^{n_{1}+1} * \Delta_{m_{2} ; y_{2}}^{n_{2}+1} * \cdots * \Delta_{m_{k} ; y_{k}}^{n_{k}+1} * f=0
$$

for each $y_{1}, y_{2}, \ldots, y_{k}$ and $z_{1}, z_{2}, \ldots, z_{k}$ in $H$. We have assumed that the exponentials $m_{i}^{\prime}(i=1,2, \ldots, s)$ are pairwise different, and similarly, the exponentials $m_{j}(j=1,2, \ldots, k)$ are pairwise different. We may assume that all these exponentials are pairwise different; otherwise we collect the equal factors in one factor in the convolution product: then the final number of all factors is not greater than $s+k$ and the corresponding powers sum up. We obtain an equation of the form

$$
\Delta_{m_{1}^{\prime \prime} ; u_{1}}^{p_{1}+1} * \Delta_{m_{2}^{\prime \prime} ; u_{2}}^{p_{2}+1} * \cdots * \Delta_{m_{t}^{\prime \prime} ; u_{t}}^{p_{t}+1} * f=0
$$

where the exponentials $m_{i}^{\prime \prime}$ are different, and $p_{1}, p_{2}, \ldots, p_{t}$ are natural numbers with $p_{1}+p_{2}+\cdots+p_{t}+t \leq d+n_{1}+n_{2}+\cdots+n_{k}+k$. For an exponential 
$m$ and a natural number $n$ let $I_{m, n}$ denote the ideal in $\mathbb{C} H$ generated by the elements $\Delta_{m ; y}^{n}$ with $y$ in $H$. Then the condition on $f$ means that

$$
I_{m_{1}^{\prime \prime}, p_{1}+1} * I_{m_{2}^{\prime \prime}, p_{2}+1} * \cdots * I_{m_{t}^{\prime \prime}, p_{t}+1} \subseteq \operatorname{Ann} \tau(f) .
$$

We show that the ideals $I_{m_{i}^{\prime \prime}, p_{i}+1}$ are pairwise co-prime for different $i$ 's. Assuming the contrary there is a maximal ideal $M$ in $\mathbb{C} H$ such that

$$
I_{m_{i}^{\prime \prime}, p_{i}+1}+I_{m_{j}^{\prime \prime}, p_{j}+1} \subseteq M
$$

for some $i \neq j$. In particular, $I_{m_{i}^{\prime \prime}, p_{i}+1}$ is contained in $M$, hence also $\Delta_{m_{i}^{\prime \prime} ; u}^{p_{i}+1}$ is in $M$ for each $u$ in $H$. As $M$ is maximal, it is prime and we infer that $\Delta_{m_{i}^{\prime \prime} ; u}$ is in $M$ for each $u$ in $H$. It follows that the ideal generated by all $\Delta_{m_{i}^{\prime \prime} ; u}$ 's with $u$ in $H$, is in $M$, too, but this ideal is exactly the maximal ideal Ann $\tau\left(m_{i}^{\prime \prime}\right)$, the annihilator of $\tau\left(m_{i}^{\prime \prime}\right)$ (see [12]). By maximality, Ann $\tau\left(m_{i}^{\prime \prime}\right)=M$, and also Ann $\tau\left(m_{j}^{\prime \prime}\right)=M$, a contradiction as $m_{i}^{\prime \prime} \neq m_{j}^{\prime \prime}$.

As the ideals $I_{m_{i}^{\prime \prime}, p_{i}+1}$ are pairwise co-prime, their product is equal to their intersection and we have

$$
I_{m_{1}^{\prime \prime}, p_{1}+1} \cap I_{m_{2}^{\prime \prime}, p_{2}+1} \cap \cdots \cap I_{m_{t}^{\prime \prime}, p_{t}+1} \subseteq \text { Ann } \tau(f),
$$

whence, by taking annihilators (see [12])

$$
\tau(f) \subseteq \sum_{i=1}^{t} \operatorname{Ann} I_{m_{i}^{\prime \prime}, p_{i}+1} .
$$

It follows that $f$ is a sum of the functions $\varphi_{i}$ with

$$
\Delta_{m_{i}^{\prime \prime} ; u}^{p_{i}+1} * \varphi_{i}=0
$$

for each $u$ in $H(i=1,2, \ldots, t)$. Using the results in [5] we infer that this equation implies

$$
\Delta_{m_{i}^{\prime \prime} ; u_{1}, u_{2}, \ldots, u_{p_{i}+1}} * \varphi_{i}=0
$$

for each $u_{1}, u_{2}, \ldots, u_{p_{i}+1}$ in $H$, hence $\varphi_{i}$ is a generalized exponential monomial of degree at most $p_{i}$ associated with the exponential $m_{i}^{\prime \prime}$ which is one of the $m_{i}^{\prime}$ 's or the $m_{j}$ 's. Our theorem is proved.

\section{Applications to a functional equation}

In [3] the authors studied the following functional equation

$$
\sum_{i=1}^{m} f_{i}\left(b_{i} x+c_{i} y\right)=\sum_{k=1}^{n} u_{k}(x) v_{k}(y),
$$

where $x$ and $y$ are in $\mathbb{R}^{d}, f_{i}, u_{k}, v_{k}$ for $1 \leq i \leq m$ and $1 \leq k \leq n$ are real functions defined on $\mathbb{R}^{d}$, and $b_{i}$ and $c_{i}$ for $1 \leq i \leq m$ are $d \times d$ real invertible matrices. They have shown that if $f_{i}$ and $v_{k}$ are continuous and the 
matrices $b_{i}^{-1} c_{i}-b_{j}^{-1} c_{j}$ are invertible for each $i \neq j$, then the $f_{i}$ 's are exponential polynomials. We shall extend these results to functional equations of the type

$$
\sum_{i=1}^{N} f_{k}\left(\varphi_{k}(x)+\psi_{k}(y)\right)=\sum_{j=1}^{d} u_{k}(x) v_{k}(y)
$$

on commutative hypergroups. As it can be expected, in this case the solutions may be generalized exponential polynomials.

We shall use the following statement, which is a slight modification of Lemma 2 in [6].

Lemma 2. Let $G$ be a finitely generated Abelian group, $f: G \rightarrow \mathbb{C}$ a function, and $V \subseteq \mathcal{C}(G)$ a finite dimensional subspace. If for each $y$ in $G$ there exists an exponential polynomial $\varphi_{y}$ such that $\delta_{y} * f-\varphi_{y}$ is in $V$, then $f$ is an exponential polynomial.

Proof. Let $G$ be generated by the elements $z_{j}$ with $j=1,2, \ldots, N$ and we may suppose that $V$ is generated by the functions $\delta_{y_{i}} * f-\varphi_{y_{i}}$ with $i=1,2, \ldots, d$, where $\varphi_{y_{i}}$ is an exponential polynomial and $y_{1}=0$. Clearly, if a subspace is invariant under the translations with these generators, then it is a variety. We define

$$
W=V+\sum_{i=1}^{d} \sum_{j=1}^{N} \tau\left(\varphi_{y_{i}+z_{j}}\right)+\sum_{i=1}^{d} \tau\left(\varphi_{y_{i}}\right) .
$$

Clearly, $W$ is a finite dimensional vector space. Moreover, $f$ is in $W$, as

$$
f=\left(\delta_{0} * f-\varphi_{0}\right)+\varphi_{0},
$$

which is in $V+\tau\left(\varphi_{0}\right) \subseteq W$. Hence it is enough to show that $W$ is translation invariant, that is, if $\varphi$ is in $W$, then $\delta_{z_{k}} * \varphi$ is in $W$ for $k=1,2, \ldots, N$. This follows from the relation

$$
\delta_{z_{k}} *\left(\delta_{y_{i}} * f-\varphi_{y_{i}}\right)=\left(\delta_{z_{k}+y_{i}} * f-\varphi_{z_{k}+y_{i}}\right)+\varphi_{z_{k}+y_{i}}-\delta_{z_{k}} * \varphi_{y_{i}} .
$$

It follows that $f$ is an exponential polynomial.

Now we can prove the following result.

Theorem 3. Let $G$ be an Abelian group, $N, d$ positive integers, and let $\varphi_{i}, \psi_{i}$ : $G \rightarrow G(i=1,2, \ldots, N)$ be automorphisms such that $\psi_{i}^{-1} \circ \varphi_{i}-\psi_{j}^{-1} \circ \varphi_{j}$ is surjective, whenever $i \neq j$. Let further $f_{i}, u_{k}, v_{k}: G \rightarrow \mathbb{C}$ be functions for $i=1,2, \ldots, N ; k=1,2, \ldots, d$ satisfying

$$
\sum_{i=1}^{N} f_{i}\left(\varphi_{i}(x)+\psi_{i}(y)\right)=\sum_{k=1}^{d} u_{k}(x) v_{k}(y)
$$

for each $x, y$ in $G$. Then all functions $f_{i}$ are generalized exponential polynomials. 
Proof. As in [3], we apply induction on $N$. For $N=1$ Eq. (3) has the form

$$
f_{1}\left(\varphi_{1}(x)+\psi_{1}(y)\right)=\sum_{k=1}^{d} u_{k}(x) v_{k}(y)
$$

for each $x, y$ in $G$. Substituting $x=\varphi_{1}^{-1}(s)$ and $y=\psi_{1}^{-1}(t)$ we have

$$
f_{1}(s+t)=\sum_{k=1}^{d} u_{k}\left(\varphi_{1}^{-1}(s)\right) v_{k}\left(\psi_{1}^{-1}(t)\right)
$$

which is the Levi-Civitá functional equation, and it is well-known that $f_{1}$ is an exponential polynomial (see e.g. [7, Chapter 10] and the references therein)

Suppose that we have proved the statement for $N$ and we consider the equation

$$
f_{1}\left(\varphi_{1}(x)+\psi_{1}(y)\right)+\sum_{i=2}^{N} f_{i}\left(\varphi_{i}(x)+\psi_{i}(y)\right)=\sum_{k=1}^{d} u_{k}(x) v_{k}(y)
$$

for $x, y$ in $G$. Let $z$ be arbitrary in $G$ and we define $w=-\psi_{1}^{-1} \circ \varphi_{1}(z)$. Then $\varphi_{1}(z)+\psi_{1}(w)=0$ and we substitute $x+z$ for $x$ and $y+w$ for $y$ in (6) to get

$$
\begin{aligned}
& f_{1}\left(\varphi_{1}(x)+\varphi_{1}(z)+\psi_{1}(y)+\psi_{1}(w)\right)+\sum_{i=2}^{N} f_{i}\left(\varphi_{i}(x)+\varphi_{i}(z)+\psi_{i}(y)+\psi_{i}(w)\right) \\
& \quad=\sum_{k=1}^{d} u_{k}(x+z) v_{k}(y+w)
\end{aligned}
$$

or

$$
\begin{aligned}
& f_{1}\left(\varphi_{1}(x)+\psi_{1}(y)\right)+\sum_{i=2}^{N} f_{i}\left(\varphi_{i}(x)+\psi_{i}(y)+\varphi_{i}(z)+\psi_{i}(w)\right) \\
& \quad=\sum_{k=1}^{d} u_{k}(x+z) v_{k}(y+w) .
\end{aligned}
$$

Subtracting Eq. (6) from the above equation we have

$$
\sum_{i=2}^{N} g_{i}\left(\varphi_{i}(x)+\psi_{i}(y)\right)=\sum_{k=1}^{d}\left[u_{k}(x+z) v_{k}(y+w)-u_{k}(x) v_{k}(y)\right],
$$

where $g_{i}(x)=f_{i}\left(x+\varphi_{i}(z)+\psi_{i}(w)\right)-f_{i}(x)$ for $i=2, \ldots, N$ and $x$ in $G$. By assumption, $g_{i}$ is a generalized exponential polynomial for $i=2,3, \ldots, N$. Observe that

$$
\varphi_{i}(z)+\psi_{i}(w)=\left[\varphi_{i}-\psi_{i} \circ \psi_{1}^{-1} \circ \varphi_{1}\right](z)=\psi_{i} \circ\left[\psi_{i}^{-1} \circ \varphi_{i}-\psi_{1}^{-1} \circ \varphi_{1}\right](z),
$$

and here $\psi_{i} \circ\left[\psi_{i}^{-1} \circ \varphi_{i}-\psi_{1}^{-1} \circ \varphi_{1}\right]$ is surjective, by assumption. Hence as $z$ runs through $G$ the values $\varphi_{i}(z)+\psi_{i}(w)$ run through $G$, too. This means 
that for $i=2$ we have that $g_{2}=\delta_{y} * f_{2}-f_{2}$ is a generalized exponential polynomial for each $y$ in $G$. In other words, for each $y$ in $G$ there is a generalized exponential polynomial $\varphi_{y}$ such that $\delta_{y} * f_{2}-\varphi_{y}$ is in the one dimensional vector space formed by the multiples of $f_{2}$. Applying Lemma 2 we get our statement. Interchanging the roles of the functions $f_{i}$ we have that each of them is a generalized exponential polynomial and our theorem is proved.

\section{Acknowledgements}

László Székelyhidi was partly supported by the Hungarian National Foundation for Scientific Research (OTKA), Grant No. K111651, and by BIUST, Botswana. Żywilla Fechner was supported by the National Science Centre, Poland, Grant No. DEC-2017/01/X/ST1/00916.

Open Access. This article is distributed under the terms of the Creative Commons Attribution 4.0 International License (http://creativecommons.org/licenses/by/4.0/), which permits unrestricted use, distribution, and reproduction in any medium, provided you give appropriate credit to the original author(s) and the source, provide a link to the Creative Commons license, and indicate if changes were made.

\section{References}

[1] Almira, J.M.: Characterization of polynomials as solutions of certain functional equations. J. Math. Anal. Appl. 459(2), 1016-1028 (2017)

[2] Almira, J.M.: Characterization of functions whose forward differences are exponential polynomials. Comment. Math. Univ. Carolin 58(4), 435-442 (2017)

[3] Almira, J.M., Shulman, E.: On certain generalizations of the Levi-Civita and Wilson functional equations. Aequ. Math. 91(1), 921-931 (2017)

[4] Bloom, W.R.: Heyer, Herbert, Harmonic Analysis of Probability Measures on Hypergroups. De Gruyter Studies in Mathematics, vol. 20. Walter de Gruyter \& Co., Berlin (1995)

[5] Djoković, D.Ž.: A representation theorem for $\left(X_{1}-1\right)\left(X_{2}-1\right) \cdots\left(X_{n}-1\right)$ and its applications. Ann. Polon. Math. 22, 189-198 (1969/1970)

[6] Shulman, E.: Decomposable functions and representations of topological semigroup. Aequ. Math. 79(1), 13-21 (2010)

[7] Székelyhidi, L.: Convolution Type Functional Equations on Topological Abelian Groups. World Scientific Publishing Co., Inc, Teaneck, NJ (1991)

[8] Székelyhidi, L.: Exponential polynomials on commutative hypergroups. Arch. Math. (Basel) 101(4), 341-347 (2013)

[9] Székelyhidi, L.: Characterization of exponential polynomials on commutative hypergroups. Ann. Funct. Anal. 5(2), 53-60 (2014)

[10] Székelyhidi, L.: Harmonic and Spectral Analysis. World Scientific Publishing Co, Pte. Ltd., Hackensack, NJ (2014)

[11] Székelyhidi, L.: A functional equation for exponential polynomials. Aequ. Math. 89(3), 821-828 (2015). https://doi.org/10.1007/s00010-014-0276-4

[12] Székelyhidi, L.: Functional equations and stability problems on hypergroups. In: Developments in Functional Equations and Related Topics, pp. 305-331 (2017) 
[13] Székelyhidi, L., Vajday, L.: Spectral analysis on commutative hypergroups. Aequ. Math. 80(1-2), 223-226 (2010)

[14] Székelyhidi, L., Vajday, L.: Spectral synthesis on commutative hypergroups. Ann. Univ. Sci. Budapest. Sect. Comput. 45, 111-117 (2016)

\author{
Żywilla Fechner \\ Institute of Mathematics \\ Łódź University of Technology \\ ul. Wólczańska 215 \\ 90-924 Łódź \\ Poland \\ e-mail: zfechner@gmail.com
}

László Székelyhidi

Department of Mathematics and Statistical Sciences

BIUST

Palapye

Botswana

e-mail: lszekelyhidi@gmail.com

and

Institute of Mathematics

University of Debrecen

Egyetem tér 1

4032 Debrecen

Hungary

Received: February 27, 2018

Revised: June 25, 2018 